\title{
Patterns of Italian high school and university students' attitudes towards physics: an analysis based on semiotic-cultural perspective
}

\author{
Italo Testa ${ }^{1}$ (D) $\cdot$ Raffaele De Luca Picione ${ }^{2} \cdot$ Umberto Scotti di Uccio $^{1}$ \\ Received: 26 February 2021 / Revised: 29 April 2021 / Accepted: 16 May 2021 / \\ Published online: 27 May 2021 \\ (C) The Author(s) 2021
}

\begin{abstract}
The purpose of this study was to analyse Italian high school and university students' attitudes towards physics using the Semiotic Cultural Psychological Theory (SCPT). In the SCPT framework, attitudes represent how individuals interpret their experience through the mediation of generalized meaning with which they are identified. A view-of-physics questionnaire was used as an instrument to collect data with 1603 high school and university students. Data were analysed through multiple correspondence analysis and cluster analysis. We identified four generalized meanings of physics: (a) interesting and important for society; (b) a quite interesting, but badly taught subject at school and not completely useful for society; (c) difficult to study and irrelevant for society; and (d) a fascinating and protective niche from society. The identified generalized meanings are significantly correlated to the choice to study physics at undergraduate level and to the choice of attending physics-related activities in high school. Implications for research are discussed.
\end{abstract}

Keywords Students' attitudes · Semiotic Cultural Psychological Theory $\cdot$ Multiple correspondence analysis $\cdot$ Cluster analysis

\section{Introduction}

Over the past 20 years, there has been an increasing concern about the students' declining interest and retention in studying physics worldwide (Arnoux et al., 2009; Eurydice, 2012; Lyons, 2006; NSB, 2014; NSF, 2017; OECD, 2008; Oon \& Subramaniam, 2011; Stokking, 2000), including Italy (Predazzi \& De Bortoli, 2005). Such disaffection is at odds with the

Italo Testa

italo.testa@unina.it

1 Department of Physics “E. Pancini”, University of Naples Federico II, Naples Italy

2 Giustino Fortunato University, Benevento Italy 
increasing demand for highly qualified personnel in the roles of research, both at academic level and in the industrial context, worldwide (EU, 2010; UN, 2012; UNESCO, 2010). Furthermore, it contributes to deepen the gap between the pervasive presence of technology in everyday life and citizens' knowledge of the physical principles upon which the used technological devices are based, with social and cultural drawbacks (AAAS, 2001; Cajas, 2001; Osborne \& Dillon, 2008). The broad social consequences of these phenomena widely motivated the large body of research on students' attitudes towards physics (Adams et al., 2006; Angell et al., 2004; Barmby \& Defty, 2006; Bøe \& Henriksen, 2013; Irving \& Sayre, 2015; Regan \& DeWitt, 2015; Reid \& Skryabina, 2002; Tytler \& Osborne, 2012, 2014; Williams et al., 2003). The attitudes towards physics may be defined as the set of feelings or, alternately, as the evaluative judgement, formed by someone about physics, capable of determining choices, decisions, etc. (Ajzen, 2001; Barmby et al., 2008; Crano \& Prislin, 2006; Kind et al., 2007; Ramsden, 1998). Hence, according to early theorists (Lemon, 1973), they bind the emotional (i.e. bad or good feelings towards physics) to the cognitive (i.e. studying physics) and behavioural spheres (i.e. the effort applied to study physics). Results of studies about students' attitudes towards physics show that physics is rated as a difficult school subject (Due, 2012; Williams et al., 2003), for which very hard work is necessary to succeed (Mujtaba \& Reiss, 2013) and expectations of good grades are lower in comparison with other sciences, as biology and chemistry (Barmby \& Defty, 2006). Moreover, physics may also be negatively perceived as abstract and scarcely useful, poorly oriented to human life and even as a possible cause of social isolation (Kessels et al., 2006; Masnick et al., 2010). These views of physics have a two-fold consequence: on the one hand, they may lead to a perceived feeling of inadequacy and lack of relevance (Eccles, 2007), thus undermining the choice of enrolling in a physics course at high school and undergraduate level (Eccles, 1983); on the other hand, abstractness, difficulty and rigor may be perceived as essential and positive features, because physics will serve to get an effective preparation for undergraduate studies and a future career in science (Carlone, 2003). Other studies proved that the intention to study physics is positively correlated with perceived future utility and personal interest (Levrini et al., 2017; Mujtaba \& Reiss, 2013; Stokking, 2000). For instance, in a study carried out in Norway, enjoyment, self-realization in physics and utility for higher education admission were amongst the factors that inspired students to choose physics at secondary school level (Bøe \& Henriksen, 2013). The authors found also that physics students at tertiary level were mostly motivated by the enjoyment of doing research in physics rather than by working with technology that involves physics. The perception of wider career choices and the interest in doing research were also amongst the factors that mostly distinguished Singapore students who chose physics at tertiary level from non-choosers (Oon \& Subramaniam, 2013). Such results suggest that students' attitudes towards physics are quite general and independent of the local context and of the educational setting.

In this paper, we argue that, in order to get a deeper understanding of the declining interest in physics at undergraduate level and in physics-related career choices, it is necessary to describe the cultural dynamics underpinning students' manifestations of representations (e.g. views, attitudes, orientations and beliefs) related to physics. On such basis, we propose to analyse the students' attitudes towards physics in the framework of the Semiotic Cultural Psychological Theory (SCPT), a socio-cultural psychological model originally aimed to describe the dynamics underpinning the manifestations of culture and in particular how one's own views shape the experience of reality. The main reason for adopting the SCPT perspective is that, at a phenomenological level, it allows to investigate how individuals differ in their 
representations, which are the implicit lenses through which the individual's relation with an object is interpreted, and in their attitude manifestations depending on the generalized meaning they share and carry out. The second reason is that the focus on sensemaking process in the SCPT will allow us to consider the individuals' personal identity and its aspects not as a static entity, but as a semiotic process of construction and negotiation in becoming (De Luca Picione, 2015; De Luca Picione \& Freda, 2016; De Luca Picione \& Valsiner, 2017; Salvatore \& Valsiner, 2010; Valsiner, 2017).

\section{Theoretical framework}

The Semiotic Cultural Psychological Theory (SCPT) is a socio-cultural psychological model (Markova, 2003; Salvatore, 2016a; Sammut et al., 2015; Valsiner, 2007), which integrates aspects of psychoanalysis (Matte Blanco, 1975; Salvatore \& Freda, 2011; Salvatore \& Zittoun, 2011), dynamic systems theory (Laura-Grotto et al., 2009; Salvatore \& Tschacher, 2012) and pragmatic semiotics (Peirce, 1992). In the SCPT theoretical perspective, psychological processes consist in sensemaking processes of interpretation that, in turn, shape experience (De Luca Picione, 2020; Salvatore et al., 2018; Valsiner, 2014). A sensemaking process is an interpretation process that links the emotional level of experience with the formal level of cognition (Salvatore \& Freda, 2011). Such process is inherently social, embodied, contextual and situated. In other words, people feel, think and act not by following context-blind universal computational rules, but by enacting sensemaking in terms of, and within the constraints of, generalized meanings, namely, prereflexive, not rationally justified assumptions concerning what the world is and how it works (Mannarini et al., 2020; Salvatore et al., 2018). Due to their implicit nature, generalized meanings are not directly observable, but, rather, they are embedded in a given cultural milieu that is the environment in which individuals' feelings, thoughts and social agency are grounded. The generalized meanings work as latent global belief systems about the self and the reality. They are recognizable only indirectly, through the cognitive and pragmatic outputs that mediate and contribute to constitute the set of cultural milieus.

To analyse a cultural milieu, the SCPT models the sensemaking process as a dynamics of sign transition within a semiotic space (Salvatore et al., 2019). In this view, a semiotic space is constituted by signs, namely "verbal statements, states of feeling, non-verbal expression, that tend to be associated over time and in so doing tend to activate a certain instance of meaning" (Salvatore et al., 2018; p. 38). The meaning does not lie in signs per se, but in the fact that one sign follows another. For instance, the statement/sign "it is a book", followed by "it is very interesting", gives relevance to the meaning that concerns the experience of reading, while the sign "it is a picture" followed by "it is very beautiful" would give relevance to the aesthetic experience in seeing a picture. As such, the sensemaking process can be viewed as a trajectory in the semiotic space that models a given cultural milieu and whose dimensions correspond to latent dimensions of sense represented by oppositional lines (e.g. "positive/negative"; "friend/ foe"; see Salvatore, 2016a; Salvatore \& Venuleo, 2013; De Luca Picione et al., 2020). SCPT postulates also that it is possible to recognize patterns around attractors that correspond to the generalized meanings of the cultural milieu by analysing the semiotic space. These attractors make a trajectory preferable with respect to a different one. Such patterns can be identified by investigating an individual's reactions to a given stimulus as, for instance, a survey about the experience of the place where she/he lives, the trustworthiness of politicians, the vision of the country's future and the perception of the role of immigrants in the society (Mannarini et al., 
2020). On such empirical base, individuals can be typified in terms of the set of generalized meanings they identify with. In other words, generalized meanings can be used for mapping and explaining variability in the individuals' attitudes embedded in a cultural milieu. In particular, on the one hand, a group of individuals can be described in terms of the set of generalized meanings that correspond to the cultural milieu in which the individuals are rooted; on the other hand, differences in views amongst groups of individuals can be explained in terms of these generalized meanings (Russo et al., 2020).

The SCPT postulates two levels of generalized meanings. The first, called symbolic universes, denotes a higher-order affect-laden system of assumptions that include views, opinions, values, beliefs and attitudes concerning facts and objects of the social and physical worlds. In other words, the symbolic universes represent generalized functions of individuals' sensemaking of the world. Symbolic universes are global and holistic, namely they include the entire experience of the relationship with the world, rather than individual parts of it (e.g. specific events, objects or domains of life). However, symbolic universes do not reflect reality; rather, each symbolic universe defines - through a process of affective semiosis - a system of values considered normative, that is, of how things are expected to go.

The second level, which we will call domain-specific generalized meanings, denotes assumptions about specific - yet generalized - domains of social and cultural life of individuals (for instance, politics, institutions, economics, science). For the purposes of this paper, we will focus on this second level of generalized meanings, taking physics as the specific domain. In particular, according to SCPT, we will model the cultural milieu of physics as a semiotic space, characterized by certain domain-specific generalized meanings. An analogy with studies in the domain of politics can be useful at this point to clarify how the SCPT framework can be applied to the physics domain. First, as each social group in a country or continent can reveal a plurality of generalized meanings in politics, so each learning community, as, e.g. high school and university students, can reveal a plurality of generalized meanings characterizing the complexity of the experience with physics. As these generalized meanings in politics are informed by a dynamic combination of factors - relational experiences, life events, economic and educational level or social class - in the same way, generalized meanings in physics are assumed to be informed by school and university experiences, performance in physics tasks, peer interactions, exposure to media communication, family background, utility value and success expectancy, etc. Moreover, as generalized meanings in politics constitute universes of sense and, as such, they influence individual political orientations, in the same way, generalized meanings in physics will be assumed to affect individuals' experience with the physical science as, for instance, the intention to choose physics at undergraduate level. Consequently, as a set of generalized meanings in politics can help understand phenomena as the rise of populism in Europe and the USA (Veltri et al., 2019), generalized meanings in physics can help understand the phenomenon of the decreasing interest in pursuing a physics career in European and US students.

\section{Research questions}

The present study seeks to identify the generalized meanings of physics characterizing a sample of Italian high school and university students and to segment the different groups of students involved in the analysis according to the generalized meanings identified. The following research questions guided the study: 
- (RQ1) Which generalized meanings of physics characterize the high school and university students involved in the study?

- (RQ2) To what extent different experiences with physics affect students' generalized meanings of physics?

\section{Methods}

\section{Participants}

The study is based on an online survey administered to a convenience sample of high school and university students. All students participated in the study on a voluntary basis. To answer our research questions, the sample was purposefully constituted by four subsamples. In the following, we report for each subsample only the number of students who completed the survey, i.e. with no missing responses (total response rate of $72 \%$ ).

Subsample 1: a proportional sample constituted by $\mathrm{N}=428$ (mean age, $21.8 \pm 1.8$ years; female students $=37.4 \%$ ) undergraduate physics students enrolled at two large universities in Italy, one located in the north of Italy and the other located in the south. This sample was chosen as representative of students who have chosen a physics career. In particular, $14.1 \%$ were physics freshman; $15.0 \%$ were sophomore students; $27.3 \%$ were students of the third year; and $43.6 \%$ had already obtained the bachelors' degree. About $60 \%$ of the respondents were from the university located in the north Italy. Gender distribution reflects that of a typical Italian cohort of physics undergraduate students.

Subsample 2: a proportional sample constituted by $\mathrm{N}=371$ (mean age, $19.8 \pm 1.6$ years; female students $=44.2 \%$ ) freshman engineering students who were attending different degree programs: electronics, telecommunication, informatics and biotechnology. This sample was chosen as representative of students who choose a STEM career, yet not directly related to physics. At the time of the data collection, students had not followed any introductory course in physics yet. Gender distribution only roughly resembles the typical constitution of an Italian cohort of engineering undergraduate students given the presence of the biotechnology freshmen.

Subsamples 3 and 4 were constituted by students from about 30 secondary schools who participated in a public engagement program at the university of the first author. The program offered to the schools was purposefully constituted by a set of two alternative extra-curricular activities: physics-focused and non-physics activities. The physics-related activities included problem-solving group sessions, practical hands-on activities and visits to research laboratories (e.g. gravitational waves). The non-physics activities included humanities and history seminars, economics and art laboratories. Approximatively each student of subsamples 3 and 4 participated to a total of about $20 \mathrm{~h}$ of extra-curricular activities. The composition of the two samples is as follows:

Subsample 3: a proportional sample, constituted by $\mathrm{N}=394$ high school students (mean age, $16.9 \pm 0.4$ years; female students $=51.5 \%$ ) who voluntarily participated in the physicsfocused extra-curricular activities. This sample was chosen as representative of students who are likely to choose a physics career or a STEM career with emphasis on physics. About $82.1 \%$ of the students attended a scientific track, which features four compulsory physics 1-h lessons per week. About $11.8 \%$ of the students attended a humanities track, which features two compulsory physics 1 -h lessons per week. About $6.1 \%$ attended a vocational track, which features no compulsory physics lessons. 
Subsample 4: a proportional sample constituted by $\mathrm{N}=369$ high school students (mean age, $17.0 \pm 0.2$ years; female students $=53.1 \%$ ) voluntarily involved in the non-physics extracurricular activities for roughly the same number of hours of subsample 3 students. This sample was chosen as representative of students who are likely not to choose a physics career or a STEM career. About $63.9 \%$ of the students attended a scientific track, $25.7 \%$ attended a humanities track, and $10.4 \%$ attended a vocational track.

\section{Instrument and measures}

The analysed dataset for this study consists in the students' responses to a 36-item Views of Physics (VOP) survey instrument. The VOP is a self-report instrument inspired to the view of context instrument used in SCPT (Ciavolino et al., 2017) and built upon previous validated instruments constituted by the following dimensions: perceived value of physics in today's society; recognition of the difficulty of physics; intrinsic interest in physics; personal relevance to work with physics; relevance of learning physics at school; recognition of physicists' role in society; and value of physicists' careers (Oon \& Subramaniam, 2013; Stokking, 2000). For each item, the students were asked to state their degree of agreement by using a 5-point Likert scale (not at all; not very; fairly; mostly; completely). Face validity of the items was assessed through discussions with local physics teachers and university instructors. The received feedbacks improved the formulation in Italian of all items.

\section{Procedure}

Subsample 1 received the VOP instrument by means of a snowball procedure which consisted in the presentation of the survey on official channels of the involved universities. The survey was approved by the physics courses coordinators in the two universities and administered during remote teaching sessions after a brief introduction.

Subsample 2 completed the VOP instrument when they voluntary applied to attend the lessons of the first-year introductory physics course. The instructions were reported at the beginning of the survey.

Subsamples 3 and 4 completed the survey online at the beginning of the planned extracurricular activities. The students received a link for completing the VOP instrument on-line, in a room with PC and tablet facilities. Instructions on how to complete the survey were given, and it was clarified that they were able to leave the survey at any time. For both groups, each school provided the e-mail contact of a teacher who was in charge to coordinate the school-university communication, keep students' records and support students in their activity. The teacher was also in charge to send to the students' families a consent document, to be signed by both parents, to participate to the research study. The ethical implications of the current research were consistent with the Ethical Code of the University of Naples Federico II (available at https:/www.unina.it/ documents/11958/14188058/DR_2425_1107\%20CODICE\%20ETICO.pdf.

\section{Data analysis}

To answer RQ1, we first performed a multiple correspondence analysis of the individuals' responses to the VOP instrument. Multiple correspondence analysis is an extension of principal component analysis to categorical variables (Abdi \& Valentin, 2007; Blasius \& Greenacre, 2006; Husson \& Josse, 2014; Le Roux \& Rouanet, 2010), and it has been used in previous studies in 
psychology field (Salvatore et al., 2018). The main reason for using the multiple correspondence analysis is that this type of analysis allows to identify patterns of associations existing between the different modalities of the items' responses. The use of this kind of analysis methodology is consistent with the major international developments of the SCPT, where a methodological pluralism is welcome in order to overcome obsolete oppositions, for example, qualitative-quantitative, idiographic-nomothetic and individual-collective (for the deepening of the issue, see De Luca Picione, 2015; Salvatore et al., 2021; Neuman, 2014; Salvatore, 2016b; Salvatore \& Valsiner, 2010; Valsiner, 2017, 2021). Moreover, for our study, a method such as the multiple correspondence analysis can operationalize in a more straightforward way the dimensions of the semiotic space that models the cultural milieu of physics in the SCPT perspective. Finally, we used this type of analysis instead of the ones adopted in previous studies about students' attitudes towards physics (Oon et al., 2020; Oon \& Subramaniam, 2013) for two further reasons. The first is related to the metric characteristics of the items, which were expressed by categorical response scales: hence, not only the distance between the categories of each scale is not linear, but also a comparison between the items' mean scores would be of little help in light of the SCPT framework. Multiple correspondence analysis addresses this metrics issue by extracting factors that are mutually orthogonal to each other (by construction) and assigning to each case a factorial score that can be interpreted as a coordinate relative to the set of active categories combined in the factor (Di Franco, 2016). Second, to obtain a simpler structure to interpret our data, as it is customary in this type of analysis, it is possible to retain only the main factorial components extracted, usually three, which are also those with a greater percentage of explained variance.

The interpretation of the extracted factorial components was based upon the contributions and test-values associated with the modalities. The contribution of a modality to a given factorial axis is the percentage of the total inertia of the factor due to the modality, while the test-value of a modality measures the statistical significance of the association between the modality and the factor, namely the probability that its distance from the centre of the factorial axis is significantly different from zero (Di Franco, 2016). Usually, for the sake of factorial components interpretation, one considers the modalities whose contribution to the factor is greater than the average contribution, calculated as 100 divided by number of active categories (Le Roux et al., 2006). Likewise, high test-values in modulus indicate a significant contribution of the corresponding modality to the factorial axis. For the present study, we first constructed the contribution test-value plot for the three factorial axes to find a threshold value for retaining the most contributing modalities according to their test-values. Then, by inspection of the graphs, we identified an estimated cut test-value for modalities with contributions greater than the average contribution (in our case, 100/180 0.55). For the first two factorial dimensions, the cut value was about $|10|$, while for the third factor, the cut value was about $|8|$. Finally, we identified the oppositional lines for the extracted factorial components using only the modalities whose associated positive (negative) test-values were above (below) the estimated cut value. Detailed results of the multiple correspondence analysis, including the contribution test-value plots and test-values tables for the three extracted factors, are reported in the Supplemental Materials.

Then, we performed a cluster analysis (CA) aimed at identifying emerging response profiles associated with different groups of individuals. The choice of adopting the CA to segment the involved students is consistent with the purpose of our analysis to detect areas of specificity in the manifestations of representations related to physics. Thus, in accordance with the SCPT framework, we consider each identified cluster as a marker of a given generalized representation of physics since it consists of a specific pattern of individual's responses that tends to appear redundantly across the sample. Amongst the possible CA methods, we used a mixed 
CA. The mixed CA first performs a $k$-means non-hierarchical procedure using as starting points randomly chosen initial centres for the clusters to obtain a first set of stable classes. Then, a Ward hierarchical method is implemented to reduce the number of stable classes as much as possible according to the principle that the fusion of two close clusters results in a minimum loss of information (Awasthi et al., 2006). The clusterization process was based on the factorial scores obtained from the multiple correspondence analysis performed on the responses to the VOP instrument. The k-means non-hierarchical procedure used as clustering criteria the similarity of the scores associated with individuals belonging to the same cluster and maximum dissimilarity of the scores associated with individuals belonging to different cluster. Therefore, to improve the initial estimation of cluster centres, all the factorial dimensions extracted (not only the three main components) were used. The subsequent nonhierarchical procedure for the optimal clustering had the following characteristics: (i) hierarchical-divisive, that is, it started from a subdivision of the sample into two main groups and then proceeded in successive steps according to a hierarchical tree structure, which was cut at a certain point to obtain the final solution; (ii) exclusive, that is, each individual cannot belong to different clusters; (iii) complete, i.e. all individuals must belong to at least one cluster; and (iv) heterogeneous, i.e. the clusters can be different in size, shape and density. Two quantitative criteria were used for choosing the number of clusters of the final solution: (1) subsequent subdivision in the dendrogram sequence produces a limited increase of the between clusters/ total inertia ratio; (2) subsequent subdivision in the dendrogram sequence produces at least one cluster with less than $5 \%$ of cases of the sample. This choice was aimed to avoid the identification of clusters with low face validity and hence harder to interpret. Both criteria allowed us to obtain a description of our sample which is not only detailed in terms of generalized meanings, but also valid from the statistical viewpoint.

The interpretation of the identified clusters was based upon the test-values of the associated modalities: high positive test-values indicate that a modality is very likely associated with the cluster, while negative t-values indicate that the modality is likely absent in the cluster. During the interpretation process, we also looked at two further indices that represent exclusivity (modal/class) and homogeneity (class/modal) of a given cluster. In particular, the modal/class percentage indicates the frequency of a modality in a given cluster over the total frequency of the same modality, while the class/modal percentage indicates the frequency of a modality with respect to all the cases in the cluster. Statistics associated to the CA analysis, including the test-values, modal/class and class/modal, are reported in the Supplemental Materials.

Finally, to answer RQ2, we first performed a one-way analysis of variance (ANOVA) to analyse if students of the four subsamples differed in their factorial scores. Then, we performed a chi-square test to inspect possible associations between the identified clusters and the four involved subsamples.

Both multiple correspondence analysis and cluster analysis were carried out by means of the package SPAD v. 5.5. The statistical analyses were carried by means of IBM SPSS v.26 package.

\section{Results}

\section{Descriptive statistics}

The descriptive statistics of the VOP instrument items are reported in Table S1 (see Supplementary Materials). Cronbach's alpha was 0.89 . 


\section{Multiple correspondence analysis}

The multiple correspondence analysis extracted 134 factorial dimensions. We report in Table S2 (see Supplementary Materials) a synthetic description of the 3 main factorial dimensions extracted. In particular, for each polarity, the first 15 items are reported. After applying the Benzécri/Greenacre simplified formula of re-evaluation, these factorial dimensions account for the $84.74 \%$ of the total inertia (respectively, $47.44 \%, 30.63 \%, 6.67 \%$ ). In consideration of the high number of factorial dimensions extracted, such percentage of variance can be considered satisfactory. Table 1 summarizes the description and interpretation of the main three factorial dimensions.

\section{Cluster analysis}

The partition in four clusters was chosen as the optimal cluster analytic solution (interclass inertia/total inertia, $0.349 / 1.028=0.339$ ). Further differentiation would not have significantly increased the interclass/total inertia ratio (e.g. ratio corresponding to five clusters, 0.388 ) or

Table 1 Interpretation of the first three factors extracted in the multiple correspondence analysis

\begin{tabular}{|c|c|c|}
\hline $\begin{array}{l}\text { Factorial } \\
\text { dimension }\end{array}$ & $\begin{array}{l}\text { Generalized } \\
\text { opposition } \\
(-\rightarrow+)\end{array}$ & Interpretation \\
\hline Factor 1 & Relevance/passivity & $\begin{array}{l}\text { The first factorial dimension polarizes two opposite, generalized, } \\
\text { affect-laden ways of signifying the field of experience with physics. On } \\
\text { the one side (polarity -), an extremely positive connotation of how } \\
\text { physics is studied at school, interesting and not abstract at all; there is } \\
\text { also a very trustworthy ideal view of physicists, lending to be engaged } \\
\text { with physics in the future; on the other side (polarity +), a not negative, } \\
\text { but neither too positive, connotation of physicists and their role in today's } \\
\text { society; there is also a not too positive view of physics as relevant and } \\
\text { interesting. We therefore interpret the factorial dimension as the marker } \\
\text { of the latent dimension of sense consisting of the generalized opposition: } \\
\text { relevance/passivity }\end{array}$ \\
\hline
\end{tabular}

Factor 2 Refusal/acceptance This factorial dimension can be interpreted as the marker of the latent dimension of sense consisting of the opposition between two very basic individuals' attitudes towards physics: refusal/acceptance. On the one side (polarity -), there is a strong negative view of physics as learned at school that lends to the demand of having nothing to do with it in the future either personally or through the physicists' work, which is disregarded and ignored. On the other side (polarity + ), physics plays an important role for solving the world demands; there is also a positive view of physicists, both in terms of their trustworthiness and job opportunities in todays' society. However, to such view does not correspond the will to pursue a career based on physics

Factor 3 Wish to be/out of The third factorial dimension is the marker of the latent dimension of sense touch of physicists' role consisting of the desire opposition: wish to be/out of touch. On the one side (polarity -), there emerges the frustrated desire of being more engaged in physics because it is quite interesting and important, but the today's society does not value physicists' work enough. On the other side (polarity + ), there is a positive view of physicists' role, careers and job opportunities, but, at the same time, physics is deemed as difficult, demanding and abstract, hence out of touch 
improved the meaningfulness of the partition. Cardinalities of emerging clusters are 661 (42.3\%), 379 (24.3\%), $102(6.5 \%)$ and $420(26.9 \%)$, respectively.

In Table S3 (see Supplementary Materials), we report a summary of the response profiles characterizing the 4 clusters (for each cluster, the most significant modalities are reported). In Table 2, we provide a brief description of response profile and our interpretation. In agreement with the framework adopted, each cluster was interpreted as the marker of a different generalized meaning of physics.

\section{Location of clusters in the semiotic space}

Figures 1 and 2 show the position of clusters/generalized meanings of physics in the semiotic space made up of the three factorial dimensions described above. For convenience, the threedimensional space is represented by two different two-dimensional subspaces, i.e. with axes (factor 1-factor 2) and (factor 1-factor 3). Clusters are labelled according to their interpretation in terms of generalized meanings.

As shown in Fig. 1, the relevance/passivity opposition line differentiates the "special value" and "blurred discipline" generalized meanings, with the former being the only generalized meaning positioned on the left side of the semiotic space, almost fully overlapping with the relevance pole. "Accepted discipline" generalized meaning is opposed to the "hard and irrelevant" generalized meaning on the refusal/acceptance direction - the former slightly associated with the acceptance polarity and the latter heavily pushed towards to pole of refusal. In turn, the opposition line wish to be/out of touch symmetrically divides "accepted discipline" from "niche discipline" and "blurred discipline" generalized meanings - the former one lightly

Table 2 Interpretation of the four identified clusters

Cluster Generalized meaning Interpretation

ID

\begin{tabular}{|c|c|c|}
\hline $\begin{array}{c}\text { Cluster } \\
1 \\
\mathrm{~N}=661 \\
(42.3 \%)\end{array}$ & Accepted discipline & $\begin{array}{l}\text { The largest cluster is characterized by a positive view of physics and physicists. } \\
\text { Physics is interesting because it intrigues, but also because it is important for } \\
\text { the development of society, both from a cultural point of view and for } \\
\text { technological applications. Society can trust the progress of physics and the } \\
\text { contribution of physicists }\end{array}$ \\
\hline $\begin{array}{c}\text { Cluster } \\
2 \\
\mathrm{~N}=379 \\
(24.3 \%)\end{array}$ & Blurred discipline & $\begin{array}{l}\text { For students in this cluster, physics seems to qualify as a discipline amongst } \\
\text { many others, unable to rise personal interest and with not too many tangible } \\
\text { effects in everyday life and society. Even in consideration of future } \\
\text { developments and employment opportunities, physics seems to be less } \\
\text { attractive than other subjects. Such attitude brings to an uncertain view of } \\
\text { physicists, with a not precise role in the society }\end{array}$ \\
\hline $\begin{array}{l}\text { Cluster } \\
3 \\
\mathrm{~N}=102 \\
(6.5 \%)\end{array}$ & $\begin{array}{l}\text { Hard and irrelevant } \\
\text { discipline }\end{array}$ & $\begin{array}{l}\text { Students in this small cluster think that physics is difficult to learn, it arises little } \\
\text { interest, and it is of little importance for society. Physicists are perceived of } \\
\text { little use to society; they are not requested by the job market and hence are } \\
\text { poorly paid. Overall, students in this cluster hold a profoundly negative and } \\
\text { stereotyped view of physics as difficult, abstract and demanding }\end{array}$ \\
\hline $\begin{array}{c}\text { Cluster } \\
4 \\
\mathrm{~N}=420 \\
(26.9 \%)\end{array}$ & Niche discipline & $\begin{array}{l}\text { This cluster is characterized by extremely positive views about physics and } \\
\text { physicists. Physics and the work of physicists are considered a fundamental } \\
\text { resource for personal life and society. Physics is neither complicated nor } \\
\text { abstract; on the contrary, its importance lies in both the theoretical aspects } \\
\text { and the technological applications. It therefore influences the daily life of } \\
\text { each of us, and it is therefore necessary to know it and a career in physics is } \\
\text { what students in this cluster are looking for }\end{array}$ \\
\hline
\end{tabular}




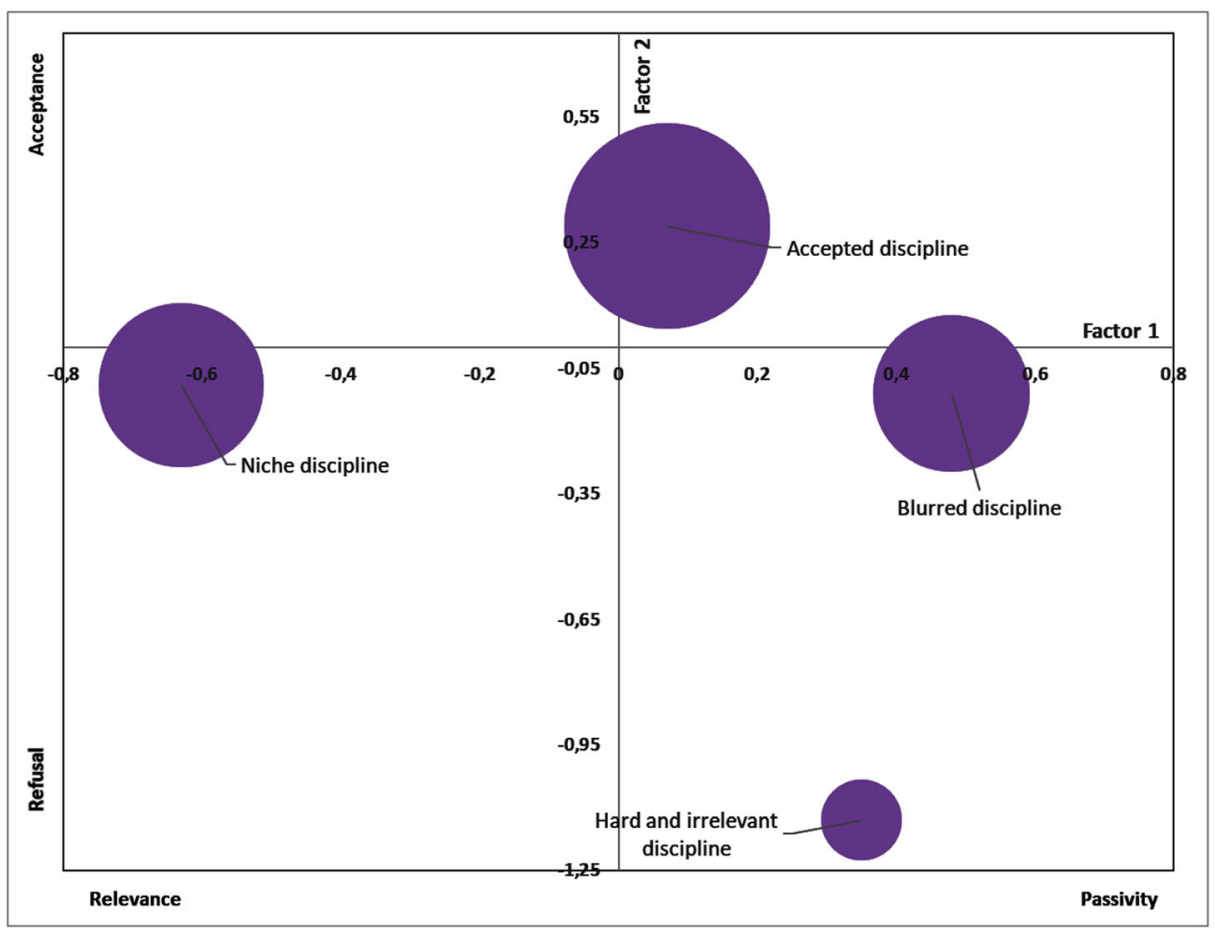

Fig. 1 Position of the students' clusters in the semiotic space identified by factor 2 ( $y$-axis) vs. factor 1 ( $x$-axis). Dimension of the dots is proportional to clusters' frequency. See Table 2 for the description of the clusters

associated with the out of touch polarity and the latter two lightly associated with the wish to be pole. "Hard and irrelevant" generalized meaning seems more polarized towards the out of touch pole (see Fig. 2).

\section{Sample segmentation}

Figure 3 reports the mean of the factorial scores of each subsample for the first three factors extracted from the multiple correspondence analysis. Since the Levene's $F$ test revealed that the homogeneity of variance assumption was not met for the relevance/passivity and refusal/ acceptance factorial dimensions $\left(\mathrm{p}<10^{-4}\right)$, we used Welch's $F$ test for these two dimensions with an alpha level of 0.05 . The one-way ANOVA shows that differences in factorial scores between the subsamples are statistically significant for both factors (Welch's $F$ (3, 860.674; $\left.844.701)>120.113, \mathrm{p}<10^{-4}, \omega^{2}>0.186\right)$. For the relevance/passivity factorial dimension, post hoc comparisons, using the Games-Howell procedure, show that all the score differences between each subsample pair are statistically significant $(\mathrm{p}<0.001)$ except that between the freshman engineering students and the high school students who voluntarily participated in the physics-focused extra-curricular activities $(\mathrm{p}=0.977)$. For the refusal/acceptance factor, the post hoc comparisons show that score differences between each subsample pair are statistically significant $(\mathrm{p}<0.002)$. For the wish to be/out of touch factorial dimension, Levene's F test was not significant $(\mathrm{p}=0.397)$. The one-way ANOVA shows that the score differences between the subsamples are statistically significant $\left(\mathrm{F}(3 ; 1558)=50.367, \mathrm{p}<10^{-4}, \omega^{2}=0.09\right)$. Post hoc comparisons, using Tuckey's HSD procedure, show that the scores of the physics students are 


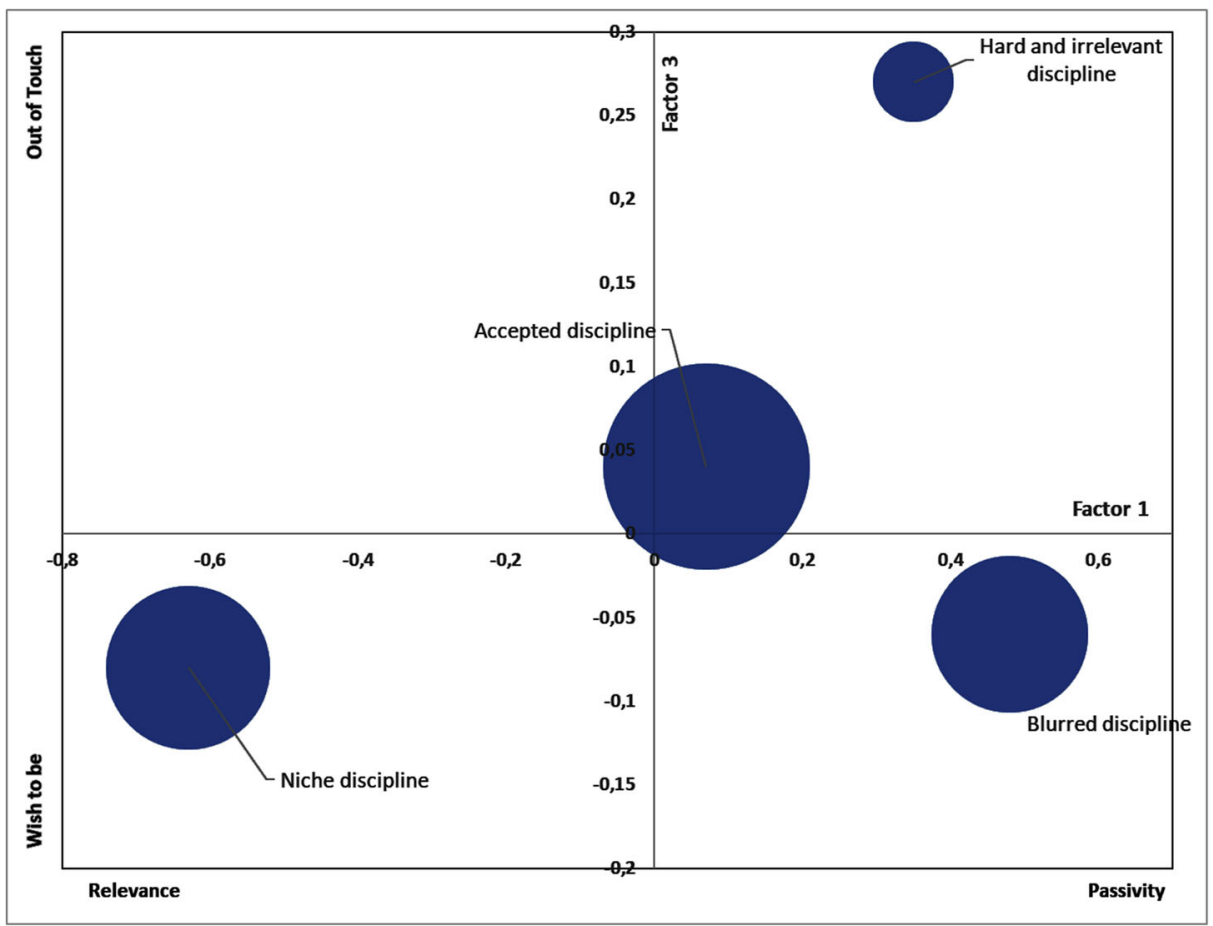

Fig. 2 Position of the clusters in the semiotic space. Factor 3 (y-axis) vs. factor 1 ( $x$-axis). Dimension of the dots is proportional to clusters' frequency. See Table 2 for the description of the clusters

significantly different from those of all the other subsamples $\left(\mathrm{p}<10^{-4}\right)$ while the differences between the scores of each pair of the remaining subsamples are not statistically different $(\mathrm{p}>0.345)$.

In Table 3, we present the association between the generalized meanings and the four subsamples involved in the study. The chi-square analysis confirms that such an association is statistically significant with a significant effect size (Cramer's V $=0.409$ ).

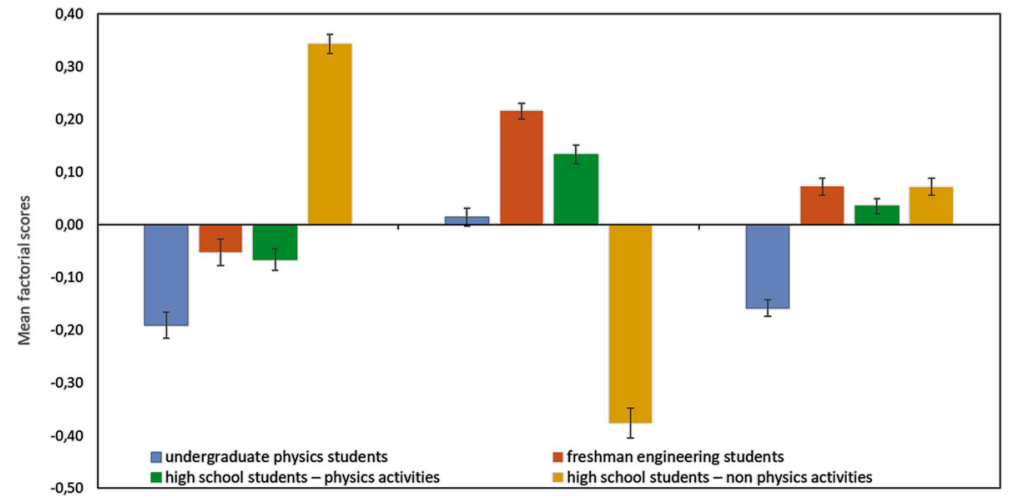

Fig. 3 Mean factorial scores of the involved subsamples for the first three extracted factors. Whiskers indicate standard errors 
Table 3 Association between generalized meanings of physics and involved subsamples

Generalized meaning of physics as discipline

\begin{tabular}{lllll} 
Subsample & Accepted (\%) & Blurred (\%) & Hard and irrelevant (\%) & Niche (\%) \\
\hline Undergraduate physics students & 23.8 & 16.1 & 4.9 & 48.8 \\
Freshman engineering students & 32.5 & 14.0 & 1.0 & 24.3 \\
High school students - physics activities & 38.1 & 11.1 & 6.9 & 22.1 \\
High school students - non-physics activities & 5.6 & 58.8 & 87.3 & 4.8 \\
Total & 100 & 100 & 100 & 100 \\
\hline
\end{tabular}

$\chi^{2}=785.781, \mathrm{df}=9, \mathrm{p}=<10^{-4}$

\section{Discussion}

There is a long tradition of studies in psychology education regarding attitudes, values, beliefs about science and physics in particular (Barmby \& Defty, 2006; Krogh \& Thomsen, 2005; Reid \& Skryabina, 2002). By adopting the SCPT perspective, this work conceptualizes these manifestations of representations in terms of the ongoing process of sensemaking - namely "a process of interpretation of the world based on emotionally charged (affect-laden) categories" (Russo et al., 2020, p. 16) when individuals deal with physics. In doing so, we modeled the cultural milieu of physics by means of a semiotic space in which the sensemaking processes substantiate in one possible trajectory around different generalized meanings. The aims of this paper were to identify these generalized meanings and to segment the different groups of students involved in the analysis according to the generalized meanings identified. In the following, we describe the extent to which we achieved the above aims.

\section{Which generalized meanings of physics characterize the high school and university students involved in the study?}

We found four generalized meanings of physics as a discipline: "accepted", "blurred", "hard and irrelevant" and "niche". These clusters/generalized meanings can be interpreted, within the SCPT, as embodied dispositions to feel and act. Therefore, they are inherently relationalnamely they do not describe the object of the relation (physics and physicists in our case) in itself, but the way the respondent perceives that physics is related to him-/herself (Tonti \& Salvatore, 2015). Hence, the identified generalized meanings can be seen as the elementary constituents of such manifestations in the sensemaking process. Thus, our study, through the application of the SCPT perspective, adds to existing body of knowledge by showing that views, attitudes, orientations and beliefs towards physics are only epiphenomena of a cultural process guided by sensemaking that shapes the way in which physics is experienced. In particular, following Salvatore et al. (2019), we can claim that each of the identified clusters embodies a particular affective meaning related to the individual experience and reflects the significance attributed to the relationships between the self, physics and physicists. In the following, we briefly discuss each of the identified cluster in light of the SCPT.

The generalized meaning "accepted discipline" is characterized by the "mostly" modalities in items explicitly referring to school experience ("I am enthusiastic when studying it", "What I learn at school affects and is relevant for daily life", "I prefer it over other subjects") and to the role of the physics professionals in everyday life ("In today's society there is great impulse in hiring them in different companies", "[Physicists] are in very high demand in today's 
society", "Relevance lies in its usefulness in solving practical problems"). Students in this cluster have a positive view of physics and physicists in society and feel committed to study physics at school or university. The "blurred discipline" generalized meaning is characterized by a reluctant sense of trust in the role of physics and physicists in society. Students in this cluster express a latent sense of unsatisfaction with the overall personal experience with physics. The passivity towards how physics is experienced leads to a lack of agency to improve the personal engagement with physics - both from the learning and utilitarian perspective - and reflects in a very weak perceived relevance. The generalized meaning "hard and irrelevant" represents a refusal reaction towards physics because it is perceived as a hard and elitist science that demands intelligence, mathematical skills and ambition to succeed (Hannover \& Kessels, 2004; Kessels et al., 2006). The refusal reaction leads an individual to see everything about physics and physicists in a negative stereotypical way (difficult, abstract, uncreative). Finally, we typified in the "niche" generalized meaning an attitude that supports a conservative, absolute, positivistic trust towards the physical science and the role of physicists in society. Students who identify with this generalized meaning are satisfied not only with how they learn physics but also with what they will do with the knowledge they will gain: in this sense, students who identify with this generalized meaning see themselves as a possible resource for the community development (Bøe \& Henriksen, 2013). Such absolute view reflects a cultural approach based on a "closed system" (Angell et al., 2004) that assumesmore or less implicitly — an inherent convergence between what students demand and what teachers provide in the classroom. Such convergence is justified by a sort of oath in which motivated students will receive what they want by motivated teachers, in order to preserve the role of physics in society as the hardest science, which always delivers right contents and drives the society in an unquestionable, efficient and effective way. In this interpretation, students' conservative attitude towards the teaching of physics may be justified by the wish to become part of a community guarded by the gatekeepers of physics (Carlone, 2003). In this sense, the notion of closed system thus acquires the meaning of niche, namely a context which is secluded from other contexts that do not share the same principles and grounds.

\section{To what extent different experiences with physics affect students' generalized meanings of physics?}

Beyond documenting the main features of the identified generalized meanings of physics, we were also interested in whether the four subsamples were significantly associated with them. The data analysis suggests a meaningful association between the nature of student's experience with physics and generalized meanings membership. In particular, the analysis of the factorial scores shows that undergraduate physics students (sample 1) are significantly polarized towards the relevance pole in the first factorial dimension and towards the wish-to-be pole in the third factorial dimension. Thus, extending previous studies (Gill \& Bell, 2013; Oon \& Subramaniam, 2013), the SCPT perspective suggests that the intention to study physics at undergraduate level is mostly related to the desire to become a physicist because of the relevance of physics from both personal and social viewpoints. Our findings suggest in particular that it is this combination that guarantees the persistence when learning physics at school and university and provides the functional support to become an appraised researcher. However, with respect to prior studies, we have found that physics undergraduates experience the world of physics and physicists as a protective niche. Such attitude of physics students may be, for instance, supported by a pervasive media, and cultural discourse that in Italy, and 
Western culture in general, detaches physics from other scientific domains (Bruun et al., 2018; Cinquini et al., 1994). Moreover, another possible reason for which these students are attracted by physics is because they do not see it as difficult and demanding, contrarily to what the majority of the rest of the students think (Bøe \& Henriksen, 2013). In a semiotic perspective, what the present study suggests is that undergraduate physics students may adopt a form of particularism and familism (Benigni \& Valsiner, 1995; Bigoni et al., 2016; Mucchi-Faina et al., 2010) characterized by a strong sense of participation and wish-to-be attitude in opposition with an external context that could not recognize the value and functional value of physics for society.

To the opposite extreme, high school students who attended non-physics activities (sample 4) tend significantly towards the passivity pole in the first factorial dimension and towards the refusal pole in the second factorial dimension. By combining this result with the evidence that the great majority of students (87\%) in the "hard and irrelevant" cluster and more than half (about 60\%) of the students in the "blurred" cluster were in group 4, our study suggests that not choosing physics is likely related to a combination of a perceived rejection by the culture of physics, for instance, due to a secluding teaching approach of physics mainly based on mathematical aspects and formula calculations (Johansson, at al., 2018) and a passive attitude towards it. Both concur to provide a sort of justification for failures in physics at school or for not wanting to be involved with physics during everyday experience. Between these extremes, the positive attitudes towards physics of sample 2 and sample 3 can be interpreted in the SCPT framework as a combination of relevance and acceptance of physics and physicists. In other word, both engineering students (sample 2) and high school students who choose physics-related activities (sample 3) are more likely to embrace the culture of physics as taught at school and the social role of physicists (Angell et al., 2004). However, this positive attitude does not necessarily imply the ambition of a career in physics, but rather the centrality of the personal relationship with physics as a value per se.

\section{Limitations}

Although it extends previous research by adopting a new perspective to analyse students' attitudes towards physics, this study has several limitations that should be acknowledged.

The first limitation concerns the choice of the VOP items. We acknowledge that the chosen items target only to a limited extent the experience of students as persons with physics. Furthermore, we acknowledge many other analyses (both quantitative and qualitative or mixed methods) can be used and carried out, mainly to investigate the subjective positioning within a cultural context and to follow semiotic trajectories of meaning (see Valsiner, 2017). Yet, adopting the wide frame of the SCPT perspective, we were interested in this study in the most prominent aspects of an individual's experience in order to infer the main characteristics of the generalized meaning with which the individual implicitly identifies. In the physics domain, we consider the generalized meanings - once identified - can help in predicting trajectories of sensemaking also for aspects of the individual's experience with physics not explicitly targeted in the VOP instrument.

The second limitation concerns the choice of the factorial dimensions used in the cluster analysis. While having retained all the factorial dimensions increased the reliability of the estimates of the initial cluster centres, we acknowledge that a different choice may have led to a different segmentation of the involved sample, with a more uniform distribution of subjects amongst the clusters. 
The third limitation concerns the use of a convenience sample. However, given the generalized valence of the identified factorial dimensions, the adoption of a non-randomized sample procedure should have negligible effect on the location of the clusters in the semiotic space, which constitutes the main focus of this work.

Fourth, the identification of the generalized meanings was based on responses by students attending different schools and curricular streams, analysed as a whole. This choice assumes that different schools and streams form a unique cultural entity. Hence, the findings could be due to a procedural artefact, rather than reflect actual features of the samples. Only further investigations with a sufficiently homogeneous sample of students can provide stronger support to our methodological choice.

The fifth limitation concerns generalizability of our results. Although our sample was large, students came prevalently from a specific region in Italy. Future research is therefore needed to examine whether similar generalized meanings and associations can be found in a national and international context.

Sixth, the VOP instrument was administered to students in the sample only once. This limits our results and does not allow us to make any inference on the stability of the identified factorial dimensions and the evolution of the generalized meanings over time. Finally, our study has a limited sample stratification. This prevents us to infer any relationships between the emerged generalized meanings and variables as gender, socio-economic status and science performance, which can have a significant impact on students' attitudes towards physics.

\section{Conclusions}

This study is the first attempt to extend the SCPT from the field of political sciences to a disciplinary area as physics, modeling its cultural milieu as a semiotic space, characterized by domain-specific generalized meanings. To identify such generalized meanings, we surveyed the attitudes towards physics of a convenience sample of high school and university students using a 36-item instrument. Collected responses were subjected to multiple correspondence analysis and cluster analysis. We found that the cultural milieu of physics is characterized by four generalized meanings, which can help explain individuals' intention to enrol in a physics course at undergraduate level or to participate to extra-curricular physics activities at high school level. Moreover, we found a significant association between the kind of student's experiences with physics and generalized meanings membership. Taken as a whole, these findings are consistent with the SCPT framework. However, more research is needed to collect further evidence that challenges the theoretical assumptions underlying the application of the SCPT perspective to the physics domain. For instance, it is worth investigating how the latent dimensions underlying the emerging representations are related to constructs as self-efficacy or confidence, which prior work has proved to influence performance, motivation and orientation towards physics. Second, longitudinal research that investigates the stability of the identified generalized representations and of the underlying factorial dimensions is critically needed. Further research can also investigate the extent to which specifically designed school and outof-school experiences can modify the underpinning dynamics of sensemaking by increasing the self-efficacy, the confidence or the performance in physics. Addressing these issues may have potential consequences for refining the SCPT-based interpretative models of students' attitudes towards physics and for devising new ways to impact on the teaching of physics at both secondary and university levels. 
Supplementary Information The online version contains supplementary material available at https://doi.org/ 10.1007/s10212-021-00563-Z.

Funding Open access funding provided by Università degli Studi di Napoli Federico II within the CRUI-CARE Agreement. This study was funded by the Italian Ministry of Education, University and Research under the national project "Piano Nazionale Lauree Scientifiche" (PLS).

Data Availability The data reported in this manuscript have been presented only in this study.

Code availability Dataset will be made available under request.

\section{Declarations}

Ethics approval The ethical implications of the current research were consistent with the Ethical Code of the University of Naples Federico II (available at https:/www.unina.it/documents/11958/14188058/DR_2425 1107\%20CODICE\%20ETICO.pdf). The work complies with relevant ethical standards for human subjects protections.

Consent to participate Students were informed about the study and had to sign a consent form to participate to the study. Those who wished not to participate to the study were allowed to not complete the survey.

Consent for publication We are aware that, if accepted for publication, a certification of authorship form will be required that all coauthors will sign.

Competing interests The authors declare no competing interests.

Open Access This article is licensed under a Creative Commons Attribution 4.0 International License, which permits use, sharing, adaptation, distribution and reproduction in any medium or format, as long as you give appropriate credit to the original author(s) and the source, provide a link to the Creative Commons licence, and indicate if changes were made. The images or other third party material in this article are included in the article's Creative Commons licence, unless indicated otherwise in a credit line to the material. If material is not included in the article's Creative Commons licence and your intended use is not permitted by statutory regulation or exceeds the permitted use, you will need to obtain permission directly from the copyright holder. To view a copy of this licence, visit http://creativecommons.org/licenses/by/4.0/.

\section{References}

Abdi, H., \& Valentin, D. (2007). Multiple correspondence analysis. In N. J. Salkind (Ed.), Encyclopaedia of Measurement and Statistics (pp. 651-657). Sage.

Adams, W. K., Perkins, K. K., Podolefsky, N. S., Dubson, M., Finkelstein, N. D., \& Wieman, C. E. (2006). New instrument for measuring student beliefs about physics and learning physics: The Colorado Learning Attitudes about Science Survey. Physical Review Special Topics - Physics Education Research, 2(1), 010101.

Ajzen I (2001) Nature and Operation of Attitudes. Annual Review of Psychology, 52 (1):27-58

American Association for the Advancement of Science. (2001). Atlas of science literacy. Washington, DC

Angell, C., Guttersrud, Ø., Henriksen, E. K., \& Isnes, A. (2004). Physics: Frightful, but fun. Pupils' and teachers' views of physics and physics teaching. Science Education, 5(88), 683-706.

Arnoux, P., Duverney, D., \& Holton, D. (2009). The rise and fall of mathematical enrolments in the French educational system: A case study. International Journal of Mathematical Education in Science and Technology, 40(1), 43-57.

Awasthi, A., Lechevallier, Y., Parent, M., \& Proth, J.-M. (2006). Using hybrid clustering to approximate fastest paths on urban networks. Journal of Data Science, 4(1), 39-65. 
Barmby, P., \& Defty, N. (2006). Secondary school pupils' perceptions of physics. Research in Science \& Technological Education, 24(2), 199-215. https://doi.org/10.1080/02635140600811585.

Barmby, P., Kind, P. M., \& Jones, K. (2008). Examining changing attitudes in secondary school science. International Journal of Science Education, 30(8), 1075-1093.

Benigni, L., \& Valsiner, J. (1995). Amoral familism and child development: Edward Banfield and the understanding of child socialization in Southern Italy. In J. Valsiner (Ed.), Child development within culturally structured environments. Comparative-Cultural and Constructivist Perspectives (Vol. 3, pp. 83-104). Ablex Publishing.

Bigoni, M., Bortolotti, S., Casari, M., Gambetta, D., \& Pancotto, F. (2016). Amoral familism, social capital, or trust? The behavioural foundations of the Italian north-south divide. The Economic Journal, 126(594), $1318-1341$.

Blasius, J., \& Greenacre, M. (2006). Multiple correspondence analysis and related methods. Multiple Correspondence Analysis and Related Methods. Chapman \& Hall/CRC.

Bøe, V. M., \& Henriksen, E. K. (2013). Love it or leave it: Norwegian students' motivations and expectations for postcompulsory physics. Science Education, 97(4), 550-573.

Bruun, M., Willoughby, S., \& Smith, J. L. (2018). Identifying the stereotypical who, what, and why of physics and biology. Physical Review Physics Education Research, 14(2), 020125.

Cajas, F. (2001). The science/technology interaction: Implications for science literacy. Journal of Research in Science Teaching, 38(7), 715-729.

Carlone, H. B. (2003). Innovative science within and against a culture of "achievement." Science Education, 87(3), 307-328

Ciavolino, E., Redd, R., Avdi, E., Falcone, F., Fini, V., Kadianaki, I., et al. (2017). Views of context. An instrument for the analysis of the cultural milieu. A first validation study. Electronic Journal of Applied Statistical Analysis, 10(2), 599-628.

Cinquini, V., Robutti, O., Vincenzi, A. B., \& Violino, P. (1994). An investigation on the effectiveness of physics teaching in Italy. International Journal of Science Education, 16(1), 45-61.

Crano, W. D., \& Prislin, R. (2006). Attitudes and persuasion. Annual Review of Psychology, 57(1), 345-374.

De Luca Picione, R. (2015). The idiographic approach in psychological research.The challenge of overcoming old distinctions without risking to homogenize.Integrative Psychological and Behavioral Science, 49(3), 360-370.https://doi.org/10.1007/s12124-015-9307-5

De Luca Picione, R. \& Freda, M. F. (2016). Borders and Modal Articulations. Semiotic Constructs of Sensemaking Processes Enabling a Fecund Dialogue Between Cultural Psychology and Clinical Psychology. Journal of Integrative Psychological and Behavioral Science, 50, 29-43. https://doi.org/10. 1007/s12124-015-9318-2.

De Luca Picione, R. \& Valsiner, J. (2017). Psychological functions of semiotic borders in sense-making: Liminality of narrative processes. Europe's Journal of Psychology, 13, 3, 532-547. https://doi.org/10. 5964/ejop.v13i3.1136.

De Luca Picione, R. (2020) The Semiotic Paradigm in Psychology. A Mature Weltanschauung for the Definition of Semiotic Mind. Integrative Psychological and Behavioral Science, 54, 639-650 https://doi.org/10.1007/ s12124-020-09555-y

De Luca Picione, R., Testa, A., \& Freda, M. F. (2020). The sensemaking process of academic inclusion experience: A semiotic research based upon the innovative narrative methodology of "upside-down-world". Human Arenas, 1-21. https://doi.org/10.1007/s42087-020-00128-4

Di Franco, G. (2016). Multiple correspondence analysis: One only or several techniques? Quality \& Quantity, 50(3), 1299-1315. https://doi.org/10.1007/s11135-015-0206-0.

Due, K. (2012). Who is the competent physics student? A study of students' positions and social interaction in small-group discussions. Cultural Studies of Science Education, 9(2), 441-459. https://doi.org/10.1007/ s11422-012-9441-z.

Eccles, J. S. (1983). Expectancies, values, and academic behaviors. In J. T. Spence (Ed.), Achievement and achievement motives: Psychological and sociological approaches (pp. 75-146). Freeman.

Eccles, J. (2007). Where are all the women? In S. J. Ceci \& W. M. Williams (Eds.), Why aren't more women in science? (pp. 199-209). American Psychological Association.

EU. (2010). Europe 2020. A European strategy for smart, sustainable and inclusive growth. European Commission.

Eurydice. (2012). Key data on education in Europe 2012. Eurydice.

Gill, T., \& Bell, J. F. (2013). What factors determine the uptake of A-level physics? International Journal of Science Education, 35(5), 753-772. https://doi.org/10.1080/09500693.2011.577843.

Hannover, B., \& Kessels, U. (2004). Self-to-prototype matching as a strategy for making academic choices. Why German high school students do not like math and science. Learning and Instruction, 14(1), 51-67. 
Husson, F., \& Josse, J. (2014). Multiple correspondence analysis. In J. Blasius \& M. Greenacre (Eds.), Visualization and verbalization of data (pp. 165-184). Chapman \& Hall/CRC.

Irving, P. W., \& Sayre, E. C. (2015). Becoming a physicist: The roles of research, mindsets, and milestones in upper-division student perceptions. Physical Review Special Topics - Physics Education Research, 11(2), 020120. https://doi.org/10.1103/PhysRevSTPER.11.020120.

Johansson, A., Andersson, S., Salminen-Karlsson, M., \& Elmgren, M. (2018). "Shut up and calculate": The available discursive positions in quantum physics courses. Cultural Studies of Science Education, 13(1), 205-226. https://doi.org/10.1007/s11422-016-9742-8.

Kessels, U., Rau, M., \& Hannover, B. (2006). What goes well with physics? Measuring and altering the image of science. British Journal of Educational Psychology, 76(4), 761-780.

Kind, P. M., Jones, K., \& Barmby, P. (2007). Developing attitudes towards science measures. International Journal of Science Education, 27(7), 871-893.

Krogh, L. B., \& Thomsen, P. V. (2005). Studying students' attitudes towards science from a cultural perspective but with a quantitative methodology: Border crossing into the physics classroom. International Journal of Science Education, 27(3), 281-302.

Laura-Grotto, R. P., Salvatore, S., Gennaro, A., \& Gelo, O. (2009). The unbearable dynamicity of psychological processes: Highlights of the psychodynamic theories. In J. Valsiner, P. Molenaar, M. Lyra, \&N. Chaudhary (Eds.), Dynamics process methodology in the social and developmental sciences (pp. 1-30). Springer.

Le Roux, B., \& Rouanet, H. (2010). Multiple correspondence analysis. Sage.

Le Roux, Borjesson, B. M., \& Bonnet, P. (2006) Performing multiple correspondence analysis (Mca) using Spad (Version 6.5). On-line, Retrieved April 2021 http://www.skeptron.uu.se/broady/sec/p-gda-0609-spadguidemca.pdf

Lemon, N. (1973). Attitudes and their measurement. B.T. Batsford Ltd..

Levrini, O., de Ambrosis, A., Hemmer, S., Laherto, A., Malgieri, M., Pantano, O., \& Tasquier, G. (2017). Understanding first-year students' curiosity and interest about physics - Lessons learned from the HOPE project. European Journal of Physics, 38(2), 025701.

Lyons, T. (2006). The puzzle of falling enrolments in physics and chemistry courses: Putting some pieces together. Research in Science Education, 36(3), 285-311.

Mannarini, T., Veltri, G. A., \& Salvatore, S. (2020). Identity, otherness, and psycho-cultural dynamics media and social representations of otherness. In T. Mannarini, G. A. Veltri, \& S. Salvatore (Eds.), Media and Social Representations of Otherness. Psycho-Social-Cultural Implications (pp. 1-16). Springer Nature Switzerland AG.

Markova, I. (2003). Dialogicality and social representations. The dynamics of mind. Cambridge University Press.

Masnick, A. M., Valenti, S. S., Cox, B. D., \& Osman, C. J. (2010). A multidimensional scaling analysis of students' attitudes about science careers. International Journal of Science Education, 32(5), 653-667.

Matte Blanco, I. (1975). The unconscious as infinite sets. An essay in bi-logic. Gerald Duckworth \& Company Ltd..

Mucchi-Faina, A., Pacilli, M. G., \& Verma, J. (2010). The two faces of familism: A cross-cultural research in India and Italy. Journal of Psychological Studies, 55(4), 365-373. https://doi.org/10.1007/s12646-0100042-1.

Mujtaba, T., \& Reiss, M. J. (2013). Inequality in experiences of physics education: Secondary school girls' and boys' perceptions of their physics education and intentions to continue with physics after the age of 16. International Journal of Science Education, 35(11), 1824-1845. https://doi.org/10.1080/09500693.2012. 762699.

National Science Board. (2014). What is the S\&E retention rate in U.S. 4-year institutions? https://www.nsf.gov/ nsb/sei/edTool/data/college-10.html, 2014.

National Science Foundation. (2017). Improving undergraduate STEM education: Education and human resources (IUSE: EHR). Retrieved from https://www.nsf.gov/pubs/2017/nsf17590/nsf17590.htm

Neuman, Y. (2014). Introduction to computational cultural psychology. Cambridge University Press.

OECD. (2008). Encouraging student interest in science and technology studies. Global Science Forum.

Oon, P. T., \& Subramaniam, R. (2011). On the declining interest in physics among students - From the perspective of teachers. International Journal of Science Education, 33(5), 727-746.

Oon, P. T., \& Subramaniam, R. (2013). Factors influencing Singapore students' choice of physics as a tertiary field of study: A Rasch analysis. International Journal of Science Education, 35(1), 86-118. https://doi.org/ 10.1080/09500693.2012.718098.

Oon, P.-T., Cheng, M. M. W., \& Wong, A. S. L. (2020). Gender differences in attitude towards science: methodology for prioritising contributing factors. International Journal of Science Education, 42(1), 89112. 
Osborne, J. \& Dillon, J. (2008). Science education in Europe: critical reflections. A report to the Nuffield Foundation

Peirce, C. S. (1992). The essential Peirce: Selected philosophical writings. In N. Houser \& C. Kloesel. (Eds.), (Vol. 1, pp. 1867-1893). Indiana University Press

Predazzi, E., \& De Bortoli, A. (2005). L'Italia e la disaffezione verso gli studi scientifici. Quaderni di Sociologia, $38,103-112$.

Ramsden, J. M. (1998). Mission impossible? Can anything be done about attitudes to science? International Journal of Science Education, 20(2), 125-137.

Regan, E., \& DeWitt, J. (2015). Attitudes, interest and factors influencing STEM enrolment behaviour: An overview of relevant literature. In E. Henriksen, J. Dillon, \& J. Ryder (Eds.), Understanding Student Participation and Choice in Science and Technology Education. Springer.

Reid, N., \& Skryabina, E. (2002). Attitudes towards physics. Research in Science and Technological Education, 20(1), 67-81. https://doi.org/10.1080/02635140220130939.

Russo, F., Mannarini, T., \& Salvatore, S. (2020). From the manifestations of culture to the underlying sensemaking process. The contribution of semiotic cultural psychology theory to the interpretation of socio-political scenario. Journal of Theory of Social Behaviour, 50, 301-320.

Salvatore, S. (2016a). The cultural psychology of desire. In J. Valsiner, G. Marsico, N. Chaudhary, T. Sato, \& V. Dazzani (Eds.), Psychology as the science of human being. The Yokohama Manifesto. Annales of theoretical psychology (pp. 33-49). Springer.

Salvatore, S. (2016b). Psychology in black and white: The project of a theory-driven science. InfoAge Publishing.

Salvatore, S., \& Freda, M. F. (2011). Affect, unconscious and sensemaking. A psychodynamic, semiotic and dialogic model. New Ideas in Psychology, 29(2), 119-135.

Salvatore, S., \& Tschacher, W. (2012). Time dependency of psychotherapeutic exchanges: The contribution of the theory of dynamic systems in analyzing process. Frontiers in Psychology, 3, 253. https://doi.org/10. 3389/fpsyg.2012.00253.

Salvatore, S., \& Valsiner, J. (2010). Between the general and the unique: Overcoming the nomothetic versus idiographic opposition. Theory \& Psychology, 20(6), 817-833. https://doi.org/10.1177/0959354310381156.

Salvatore, S., \& Venuleo, C. (2013). Field and dynamic nature of sensemaking. Theoretical and methodological implications. Papers on Social Representations. Special Issue Semiotics and Social Representations: a mutual cultivation, 22, 21, 1-21, 41. Retrieved July 2020, http://psr.iscte-iul.pt/index.php/PSR/article/view/ $310 / 270$

Salvatore, S., \& Zittoun, T. (2011). Outlines of a psychoanalytically informed cultural psychology. In S. Salvatore \& T. Zittoun (Eds.), Cultural Psychology and Psychoanalysis in Dialogue. Issues for Constructive Theoretical and Methodological Synergies (pp. 3-46). Information Age.

Salvatore, S., Fini, V., Mannarini, T., Veltri, G. A., Avdi, E., Battaglia, F., et al. (2018). Symbolic universes between present and future of Europe. First results of the map of European societies' cultural milieu. PLoS ONE, 13(1), e0189885. https://doi.org/10.1371/journal.pone.0189885.

Salvatore, S., Valsiner, J., \& Veltri, G. A. (2019). The theoretical and methodological framework. Semiotic cultural psychology, symbolic universes and lines of semiotic forces. In S. Salvatore, V. Fini, T. Mannarini, J. Valsiner, \& G. A. Veltri (Eds.), Symbolic Universes in Time of (Post)Crisis. The Future of European Societies (pp. 24-49). Springer.

Salvatore, S., De Luca Picione, R., Cozzolino, M., Bochiccio, V., \& Palmieri, A.(2021). The role of affective sensemaking in the constitution of experience. Integrative psychological and behavioral science.https://oi. org/10.1007/s12124-020-09590-9.

Sammut, G., Andreouli, E., Gaskell, G., \& Valsiner, J. (2015). Social representations: A revolutionary paradigm? In G. Sammut, E. Andreouli, G. Gaskell, \& J. Valsiner (Eds.), The Cambridge Handbook of Social Representations, Cambridge Handbooks in Psychology (pp. 3-11). Cambridge University Press. https:// doi.org/10.1017/CBO9781107323650.003.

Stokking, K. M. (2000). Predicting the choice of physics in secondary education. International Journal of Science Education, 22(12), 1261-1283.

Tonti, M., \& Salvatore, S. (2015). The homogenization of classification functions measurement (HOCFUN): A method for measuring the salience of emotional arousal in thinking. American Journal of Psychology, $128(4), 469-483$.

Tytler, R., \& Osborne, J. (2012). Student attitudes and aspirations towards science. In B. J. Fraser, K. G. Tobin, \& C. J. McRobbie (Eds.), Second international handbook of science education (pp. 597-625). Springer.

Tytler, R., \& Osborne, J. (2014). Attitudes, identity, and aspirations toward science. In N. G. Lederman \& S. K. Abell (Eds.), Handbook of research on science education (Vol. II, pp. 82-103). Routledge. 
UNESCO. (2010). Engineering: Issues, challenges and opportunities for development. United Nations Educational, Scientific and Cultural Organization.

United Nations. (2012). Millennium development goals. www.un.org/millenniumgoals/

Valsiner, J. (2007). Culture in minds and societies. Foundations of cultural psychology. Sage.

Valsiner, J. (2014). An invitation to cultural psychology. Sage.

Valsiner, J. (2017). From methodology to methods in human psychology. Springer.

Valsiner, J. (2021). General human psychology: foundations for a science. Springer.

Veltri, G. A., Redd, R., Mannarini, T., \& Salvatore, S. (2019). The identity of Brexit: A cultural psychology analysis. Journal of Community \& Applied Social Psychology, 29(1), 18-31. https://doi.org/10.1002/casp. 2378.

Williams, C., Stanisstreet, M., Spall, K., Boyes, E., \& Dickson, D. (2003). Why aren't secondary students interested in physics. Physics Education, 38(4), 324-329.

Publisher's note Springer Nature remains neutral with regard to jurisdictional claims in published maps and institutional affiliations.

\title{
Authors' Current Themes of Research
}

Students' attitudes, science identity, subjectivity, affectivity, processes of sensemaking in education

\section{Authors' Most Relevant Publications in the Field of Psychology of Education}

De Luca Picione, R., Testa, A., \& Freda, M. F. (2020). The sensemaking process of academic inclusion experience: A semiotic research based upon the innovative narrative methodology of "upside-down-world". Human Arenas, 1-21. 10.1007/s42087-020-00128-4

Esposito, G., Freda, M.F., De Luca Picione, R. (2016). Reflexivity or "reflexivities" in higher education: Conceptualizing unique reflexive process. In: Freda, M.F., Gonzàlez-Monteagudo, J., Esposito, G. (2016) (Eds). Working with Underachieving Students in Higher Education: Fostering Inclusion through Narration and Reflexivity. pp.32-42. Abingdon: Oxon; New York: NY; Routledge

Testa I., Colantonio A., Galano S., Marzoli I., Trani F., \& Scotti di Uccio U. (2020) Effects of instruction on students' overconfidence in introductory quantum mechanics. Phys. Rev. Phys. Educ. Res. 16, 010143

\author{
Address of the authors \\ - Italo Testa, Umberto Scotti di Uccio \\ Department of Physics "E. Pancini”, University of Naples Federico II, Italy \\ Complesso M.S Angelo, Via Cintia \\ 80126 Naples, Italy \\ - Raffaele De Luca Picione \\ Giustino Fortunato University \\ Viale Raffaele Delcogliano, 12, \\ 82100 Benevento, Italy
}

e-mail address of the corresponding author

italo.testa@unina.it

Italo Testa (https://orcid.org/0000-0002-8655-683X), $\mathrm{PhD}$, is an associate professor of Physics Education at "Ettore Pancini" Physics department of the University of Naples Federico II. His current research interests include the design of inquiry-based teaching-learning sequences at secondary school level, quantitative methods to assess students' learning in and attitudes towards physics, students' interpretation of visual representations in astronomy and physics teachers' professional development.

Raffaele De Luca Picione (https://orcid.org/0000-0002-5951-8336), PhD, is an associate professor of Dynamic Psychology at the Giustino Fortunato University (Benevento, Italy) and psychotherapist with a psychoanalytic training. His research focuses on subjectivity, affectivity, modeling of the mind and semiotic borders, temporality and processes of sensemaking, starting from a psychoanalytic and semiotic perspective. 
Umberto Scotti di Uccio Umberto Scotti di Uccio, PhD, is an associate professor of Physics of Solid State at "Ettore Pancini" Physics department of the University of Naples Federico II. His current research interests concern the development of teaching-leaning sequences in quantum physics, context-based inquiry approaches and physics teachers' professional development. 